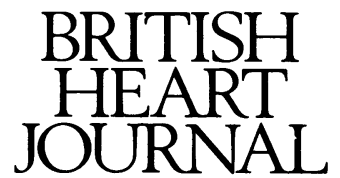

\title{
Getting a handle on the prevalence of coronary heart disease
}

Few scientists are as skilled as epidemiologists at tormenting clinicians. The practice began when John Snow, an academic ruffian, damaged physicians' pride and public property by removing the handle of the Broad Street pump during a cholera epidemic.

In this issue of the British Heart Journal the contretemps continues ( $\mathrm{p}$ 295). Smith et al confirm the high prevalence of coronary heart disease in Scotland and establish that there is considerable variation in disease prevalence among Scottish districts. This observation extends the earlier work of the Scottish Heart Health Study which showed regional differences in the death rate from coronary heart disease and in the prevalence of risk factors for ischaemia. ${ }^{1-4}$ Understanding these variations in a country with one of the highest rates of coronary heart disease in the world may lead to a better understanding of preventable causes of coronary heart disease.

The difficulty that clinicians and epidemiologists have in communicating is illustrated by this paper and primarily centres on the methods used by Smith et al who estimate the prevalence of coronary heart disease. Unlike the "hard" data for rates of hospital admissions for infarction, prevalence is difficult to measure accurately and must be estimated by symptoms, by abnormalities in the electrocardiogram, or by other more specific tests. The Scottish Heart Health Study used several methods to estimate disease prevalence, including a self administrated Rose questionnaire for angina pectoris, a resting electrocardiogram coded by the Minnesota criteria for $Q / Q S$ patterns for past transmural infarction and by $S T$ and $T$ wave abnormalities for resting ischaemia, and a self-reported history of coronary heart disease. These are accepted techniques with good, population based, repeatability and validity for the detection of coronary heart disease. But they are also commonly used clinical tests that clinicians, for good reason, do not trust to diagnose coronary heart disease accurately in individual patients.

Because these methods are standardised they can be used to estimate disease prevalence in different large populations. Their limited sensitivity and specificity are outweighed by their repeatability when applied to the same group of people, their ease of use, and their proven validity in identifying groups of people at risk of developing the consequences of coronary heart disease. The methods work particularly well for testing hypotheses about associations between coronary heart disease and its potential causes because random misclassification of an individual as having or not having coronary heart disease tends to obscure true associations but not create false associations. For comparisons between different groups, random errors and even biases leading to overestimates or underestimates of the actual number of true cases of disease are less critical because the comparison is the measure of interest. Moreover, the use of all three methods allows the estimates by each method to be compared.

But the extent to which these methods can be applied to smaller groups - for example, to a Scottish district or to an individual-is unclear. For small groups of people and certainly for individual patients we need more sensitive and specific methods. For example, it would be ludicrous to label an individual as having coronary heart disease on the basis of their answers to the Rose angina questionnaire or $S T$ and $T$ wave abnormalities on their resting electrocardiogram.

Even the coded resting electrocardiogram, one of the more objective methods of assessing disease prevalence, is seriously flawed. Old myocardial infarctions can cause $Q$ or QS waves in the electrocardiogram, and $Q$ waves from an infarction may resolve. The relation between coronary heart disease and ST and $\mathrm{T}$ wave changes on the resting electrocardiogram is even less precise because these repolarisation abnormalities are likely to be caused by left ventricular hypertrophy or other commonly occurring cardiac processes.

A patient's self-reported history of a previous infarction or diagnosis of angina is also not specific for diagnosing ischaemic heart disease. Even for carefully recorded data, verification of self-reported history is not perfect. For example, a diagnosis of myocardial infarction can be confirmed in hospital records in only $85 \%$ of those with a self-reported diagnosis. ${ }^{5}$ The validation of less definite end points is probably much less consistent.

How do these limitations in survey methods affect the results of the Scottish Heart Study? There seems to be reasonable agreement between these methods, and reasonable correlations with measures of atherosclerotic heart disease-such as the category specific death rates. The evidence suggests that there are regional differences in the prevalence of coronary heart disease in Scotland. However, Smith et al go beyond this important observation to imply that these methods can be relied upon to support the view that coronary heart disease is more prevalent in women than in men.

Data derived from the Rose questionnaire, but not from the self-reported past medical history or the coded electrocardiogram, indicated a higher prevalence of angina (particularly the milder forms) in women. Other studies consistently found a high rate of mild chest pain in women. ${ }^{6}$ We do not know whether this difference reflects a peculiarity of the Rose questionnaire or a difference in the perception or description of chest pain between sexes.

Many physicians assume that women report a high rate 
of angina in response to the Rose questionnaire because the questionnaire lacks specificity and because women are describing a hypersensitivity to pain or anxiety related symptoms. But which estimate of disease prevalence is correct? Could men be underreporting their symptoms? These are important questions because there is a growing awareness that coronary heart disease in women may be misdiagnosed or undertreated and that this may account for their increased morbidity after interventions such as coronary artery surgery and angioplasty have been delayed. ${ }^{7}$

That at least part of the answer lies in the limitations of the Rose questionnaire or in a difference in the pain threshold of men and women in the Scottish Heart Health Study is suggested by the lack of correlation with other measures of ischaemic heart disease and the observation that women had a lower rate of more severe angina, a lower rate of $S T$ and $T$ wave changes and of $Q$ wave changes, and a lower rate of ischaemic heart disease diagnosed clinically. This point needs further exploration.

As this story unfolds and we learn more about regional differences in the prevalence of ischaemic heart disease as well as differences in presentation in men and women, physicians should bear in mind that the Rose questionnaire, Minnesota coded electrocardiograms, and validated patient histories are all reasonably good survey methods. Epidemiologists should remember the major limitations of these measures when they are applied to small populations and to subgroups of a population. They should be cautious before they recommend the extrapolation of such data to policy recommendations for changes in clinical practice or in resource allocation.

Despite the bluster and noise that they may make, clinicians rely on epidemiologists for leadership. Epidemiological observations can have direct and immediate effects on the type of practice allowed or recommended for individual patients in whom atherosclerotic heart disease is suspected. The implication that physicians are systematically misdiagnosing women or any subpopulation is taken as a personal affront by most physicians. It is irresponsible for epidemiologists to present incomplete and not fully developed observations and theories because publication of such ideas in a clinical journal implies that they should be incorporated into clinical practice. In addition, there is concern that these simple tests used in population research will be forced on physicians as necessary or sufficient screening procedures for the diagnosis or treatment of individual patients with suspected coronary artery disease.

Physicians are a tolerant lot. They can probably come to terms with the knowledge that the west of Scotland has more ischaemic heart disease than the east. And as research continues into the epidemiology of coronary heart disease, they can perhaps accept the incongruous finding that young women have a higher rate of angina despite lower mortality and a lower infarction rate than young men.

Division of Cardiology,

Department of Medicine,

University of North Carolina,

Chapel Hill, North Carolina, USA

Department of Epidemiology,

School of Public Health,

University of North Carolina,

Chapel Hill, North Carolina, USA

ROSS J SIMPSON, Jr

1 Crombie IK, Smith WCS, Kenicer MB, Tunstall-Pedoe H. Geographical variation in coronary heart disease mortality in Scotland. Health Bull (Edinb) 1986;44:193-202.

2 Smith WCS, Tunstall-Pedoe H, Crombie IK, Tavendale R. Concomitants of excess coronary deaths - major risk factor and lifestyle findings from 10,359 men and women in the Scottish Heart Health Study. Scott Med J $1989 ; 34: 550-5$

3 Tunstall-Pedoe H, Smith WCS, Crombie IK, Tavendale R. Coronary risk factor and lifestyle variation across Scotland: results from the Scottish

Heart Health Study. Scott Med J 1989;34:556-60.
Crombie IK, Smith WCS, Tavendale R, Tunstall-Pedoe H. Geographical clustering of risk factors and lifestyle for coronary heart disease in the Scottish Heart Health Study. Br Heart J 1990;64:199-203.

5 Schiedt-Nave C, Barrett-Connor E, Wingard DL. Resting electrocardiographic abnormalities suggestive of asymptomatic ischemic heart disease associated with non-insulin-dependent diabetes mellitus in a defined population. Circulation 1990;81:899-906.

6 Wilcosky T, Harris R, Weissfeld $L$. The prevalence and correlates of Rose questionnaire angina among women and men in the lipid research clinics program prevalence study population. Am J Epidemiol 1987;125:400-9.

7 Khan SS, Nessim S, Gray R, Czer LS, Chaux A, Matloff J. Increased mortality of women in coronary artery bypass surgery: evidence for referral bias. Ann Intern Med 1990;112:561-7. 